\title{
El presente de subjuntivo con valor retrospectivo en la prensa peruana
}

\section{The Present Subjunctive with Retrospective Value in the Peruvian Press}

\author{
Mabel Alelí Castro Yauri \\ Universidad Nacional Mayor de San Marcos, Lima, Perú \\ https://orcid.org/oooo-0002-9379-8992 \\ mabel.castro@unmsm.edu.pe
}

\begin{abstract}
Resumen
El presente artículo analiza el empleo del presente de subjuntivo para expresar eventos pasados en las publicaciones periodísticas de diarios digitales peruanos. El objetivo de la investigación consiste en determinar si el favorecimiento del presente de subjuntivo en la prensa es influenciado por variables extralingüísticas como el género discursivo y el registro. Los datos han sido obtenidos de 120 textos digitales de diarios digitales serios y 30 textos de diarios chichas. A partir de un análisis probabilístico, se concluye que la variable significativa estadísticamente es el género discursivo. Además, se identifica a los reportajes informativos y las noticias como los textos que incrementan la probabilidad del uso del presente de subjuntivo.
\end{abstract}

Palabras clave: presente de subjuntivo, pretérito imperfecto, prensa digital, género discursivo, registro

\begin{abstract}
This article analyzes the use of the present subjunctive to express past events in journalistic publications of Peruvian digital newspapers. The aim of the research is to determine whether the favoring of the present subjunctive in the press is influenced by extralinguistic variables such as discursive genre and register. The data have been obtained from 120 digital texts of serious digital newspapers and 30 texts of digital «chicha» newspapers. From a probabilistic analysis, it is concluded that the statistically significant variable is the discursive genre. In addition, informative reports and news are identified as the texts that increase the probability of the use of the present subjunctive.
\end{abstract}

Keywords: present subjunctive, past imperfect, digital press, discursive genre, register. 
El presente de subjuntivo con valor retrospectivo en la prensa peruana

\section{Introducción}

El presente de subjuntivo (cante) y el pretérito imperfecto (cantara,-se), en algunas variedades del español americano, actúan como variantes morfosintácticas. En ciertos contextos discursivos, en los que se espera el uso del pretérito imperfecto, el hablante opta por el presente. Una muestra de ello se observa en enunciados como los siguientes: ¿Qué hizo que Alan tome la decisión de dispararse en la cabeza?; Recuerdo mucho que buscabas un editor que te apoye en la edición con las gráficas; Los policías llegaron antes que se escuchen los disparos. En estos casos, la elección del presente en lugar del pretérito imperfecto (tomara, apoyara, escucharan) conlleva a que el evento remitido por el verbo se interprete como un hecho pasado (Rojo y Veiga, 1999; Arrizabalaga, 2009; Sessarego, 2008).

En este caso de variación, el presente de subjuntivo constituye la forma no esperada; puesto que, en el sistema verbal español, los contenidos temporales del presente de subjuntivo son el 'presente' y el 'futuro' (RAE, 2010). Este uso particular del presente ha sido descrito por la RAE (2010) como un fenómeno popular, propio del español andino, y ha sido registrado en países como México, Venezuela, Argentina, Perú, Bolivia, Ecuador y Chile (Arrizabalaga, 2009; Sessarego, 2008).

La variación en el español peruano ha sido estudiada, entre otros, por Arrizabalaga (2009) y Sessarego (2008). El trabajo del primero demuestra la ocurrencia y la extensión del fenómeno en esta variedad. Arrizabalaga reporta casos de la prensa nacional, del habla espontánea, y del habla culta de Lima. A partir de estos datos, sostiene que la alternancia se manifiesta de manera amplia en la prensa. Además, reconoce tres tipos de estructuras sintácticas en los que la variación sucede: las subordinadas completivas, las finales y las relativas.

Por su parte, el estudio de Sessarego (2008) se centra en establecer los factores lingüísticos que favorecen y desfavorecen la elección del presente por el pretérito imperfecto en el español peruano y en el boliviano. Con este objetivo, analiza 350 oraciones nominales procedentes de textos periodísticos disponibles en el Corpus de Referencia del Español Actual (CREA). Luego, calcula la probabilidad de la ocurrencia del presente de subjuntivo según el tipo de verbo de la oración principal (verbos de creación/otros), el tiempo del verbo principal (pretérito/otros), y la agentividad de la oración subordinada (agente/no agente). Para los casos del español peruano, determina que los verbos de creación limitan el uso del presente y que las subordinadas sin agente lo favorecen. Asimismo, establece que, entre ambas variables, la más fuerte es la primera. 
Los trabajos de Arrizabalaga y Sessarego revelan que el uso del presente de subjuntivo para aludir a hechos pasados (el incumplimiento de la concordancia) no es propio del habla popular. Ambos autores recopilan casos de la prensa peruana, lo cual muestra que la variante no estándar se ha extendido a un contexto más formal, en el que las producciones suponen un mayor grado de planificación en relación con el habla espontánea. No obstante, ninguno de ellos estudia la ocurrencia del presente de subjuntivo en este tipo de textos.

Partiendo del hecho de que el lenguaje en la escritura periodística puede variar dependiendo de factores situacionales como el género periodístico, el tema, el tipo de diario o la sección (Vellón Lahoz, 2011), la cuestión que nos planteamos es si el tipo de género discursivo y el registro explican la ocurrencia del presente de subjuntivo en los diarios digitales peruanos. Por tanto, el objetivo del estudio es determinar si ambas variables son significativas estadísticamente. Con este fin, el análisis parte de dos objetivos específicos: establecer si (a) los diarios menos formales y (b) las noticias favorecen la ocurrencia del presente de subjuntivo con valor retrospectivo.

Las conclusiones a las que se arriba pueden ser tomadas como una contribución a los estudios del español peruano; puesto que, complementa lo que hasta el momento se conoce sobre la variación en cuestión. Los datos de la prensa y su cuantificación pueden tomarse como evidencia para estudios futuros que busquen evaluar el desarrollo de la variante innovadora (presente de subjuntivo) en esta variedad lingüística, es decir, que busquen determinar si forma parte o no de la norma.

El contenido del presente artículo se organiza de la siguiente manera. En primer lugar, se describe el marco teórico. Este incluye la descripción de los tiempos del presente de subjuntivo y del pretérito imperfecto en el sistema verbal español, así como la caracterización del género discursivo, de los géneros periodísticos, del registro y de los tipos de diarios digitales peruanos. En segundo lugar, se detalla el método de la recolección y del análisis de los datos. En tercer lugar, se expone los resultados del análisis estadístico descriptivo y probabilístico, y la discusión de los mismos. En cuarto lugar, se presentan las conclusiones del estudio. 


\section{Marco teórico}

\subsection{Los tiempos del pretérito imperfecto y del presente de subjuntivo}

El pretérito imperfecto (cantara,-se) y el presente (cante) constituyen las formas simples de los verbos de subjuntivo. Cada una cumple funciones temporales distintas, adicionales a las que denotan sus nombres. El presente de subjuntivo contiene dos valores: el 'presente' (me molesta que cojas mi celular) y el 'futuro' (espero que lleguen por mí, o me quedaré a dormir). Ambas funciones expresan relaciones temporales cuyo punto de referencia es el momento de la comunicación (Rojo y Veiga, 1999). El tiempo presente denota un hecho simultáneo al tiempo en que en se produce el enunciado. En cambio, el tiempo futuro indica que un evento es posterior a este. Debido a esta diferencia, en el primer ejemplo, cojas se interpreta como un hecho que ocurre repetidamente. En el segundo, por el contrario, la acción denotada por lleguen aún no ha sido llevada a cabo.

Los tiempos del pretérito imperfecto, de otro lado, han sido clasificados en dos grupos por Rojo y Veiga (1999) y Veiga $(1996,2014)$. Para Rojo y Veiga, las realizaciones temporales del pretérito imperfecto se dividen en básicas y dislocadas. Veiga, por su parte, distingue entre un pretérito imperfecto irreal y uno no irreal; y enumera los tiempos en cada caso. Aunque la denominación de los grupos varía en cada referencia, el criterio se fundamenta en un mismo hecho. El pretérito imperfecto, de acuerdo con los autores, puede expresar un matiz modal de irrealidad, adicional al de modo subjuntivo. Este contenido se interpreta como la negación implícita del hecho expresado por el verbo subjuntivo, como en ojalá vivieras en Lima (= no vives en Lima); o en «si me escucharas» (=no me escuchas). Esta negación no se infiere en me encantó que vinieras a casa (=viniste a casa), o en me pidió que llegara temprano (=llegaría temprano). Los tiempos del pretérito imperfecto entonces varían dependiendo de la ausencia o la presencia de este contenido modal.

En cuanto forma subjuntiva no irreal, el pretérito imperfecto puede expresar tres valores temporales: 'pretérito', 'copretérito' y 'pospretérito' (Veiga, 1996 y 2014). En función de pretérito, el verbo manifiesta que el evento sucedió en un tiempo anterior al momento de la comunicación (Rojo y Veiga, 1999). En no creo que visitaras a tu abuela, se interpreta que la acción ya ha sido llevada a cabo. De otro lado, cuando su valor temporal es el de 'copretérito', el verbo indica que una acción (o una situación) es simultánea a un hecho pasado (Rojo y Veiga, 1999).

148 Lengua y Sociedad. Revista de Lingüística Teórica y Aplicada 
Esta definición se evidencia en los siguientes casos: jugué por horas sin que nada me distrajese, y me alegré de que Erika viviera en mi casa. Del primer enunciado, se interpreta que mientras jugaba, no me distraje; y del segundo, se entiende que, en el momento en que me alegré, Erika estaba viviendo en mi casa.

Por último, cuando funciona como 'pospretérito', el pretérito imperfecto señala que una acción es posterior a un evento pasado (Rojo y Veiga, 1999). Esta definición se muestra en los siguientes casos: esperaba que Andrea llegara, y le rogó a Pedro que viajara a México. Se interpreta en el primer ejemplo que Andrea no había llegado aún; y en el segundo, que cuando se lo pidieron, Pedro no había viajado aún.

\subsection{El registro}

Moreno Fernández (1998) define el estilo — no emplea el término registro- como los usos lingüísticos que dependen de la situación y el contexto de la comunicación. Estos estilos pueden variar de lo más a lo menos formal. La gradualidad, como característica de la variación lingüística, responde al hecho de que una misma situación comunicativa puede tender a una mayor o menor formalidad. Por ello, sostiene que no se pueden identificar los registros como unidades discretas, sino como un continuo, una escala en el que los usos se distribuyen en puntos intermedios o extremos. Asimismo, sostiene que el grado de formalidad de un discurso depende de los diferentes aspectos que supone un proceso comunicativo, tales como el hablante, el interlocutor, el medio, la situación comunicativa, entre otros. De acuerdo con el autor, cada uno de estos factores, y la interrelación entre estos, tendrá un peso distinto en el momento que el hablante adopte un estilo formal o informal.

Estos factores son divididos por el autor en dos grupos: los personales y los no personales. En el primero ubica al hablante y al oyente. Por un lado, señala que las estrategias discursivas, o el nivel de consciencia sobre su producción son aspectos del hablante que influyen en la producción de un discurso más formal o menos formal; por el otro, que el tipo de relación entre el oyente y el hablante mantiene en el momento concreto de la comunicación (poder/solidaridad) también será un factor decisivo. En el segundo grupo, los factores no personales, identifica al discurso y al contexto. Los elementos del discurso que influyen en la variación del estilo, para el autor, son el tema y el tipo de comunicación. En esta última categoría menciona como modalidades básicas de la comunicación oral, la conversación y 
El presente de subjuntivo con valor retrospectivo en la prensa peruana

Mabel Alelí Castro Yauri

el discurso monologado. Por último, reconoce como componentes del contexto, el lugar, el momento y la actividad desarrollada.

\subsection{El género discursivo y los géneros periodísticos}

El género discursivo, desde el enfoque de la Escuela de Sidney, se define como un evento comunicativo que posee una estructura establecida socialmente, a través del cual, los hablantes concretan un propósito (Martin-Martin, 2003; Bawarshi y Reiff, 2010). En este sentido, la estructura de un género consta de etapas o instancias que cumplen funciones comunicativas, cada cual orientada a llevar a cabo el objetivo general que caracteriza al género. Ejemplo de géneros son las cartas, las noticias, los artículos de investigación, las entrevistas, etc.

En el periodismo existe una diversidad de géneros que han sido clasificados, principalmente, según el propósito del quehacer periodístico: informar, interpretar y opinar (Mejía Chiang, 2012). Las noticias, los reportajes, las columnas de opinión, los editoriales, las entrevistas, ente otros, han sido clasificados teniendo en consideración el objetivo de cada tipo de texto; puesto que los contenidos que se permiten, el modo en que se presentan y el uso del lenguaje en cada género están supeditados a la función general de cada uno de ellos.

En este estudio presentamos las características generales de las noticias, los reportajes informativos, los interpretativos y las columnas de opinión sin tener en consideración un marco conceptual específico:

\subsubsection{Las noticias}

Paniagua Santamaría (2009) distingue dos sentidos de la palabra noticia. En el primer sentido, la noticia es un hecho o un acontecimiento y, en el segundo sentido, la noticia es un texto. En esta línea, Alex Grijelmo (2014) señala que un hecho es considerado noticia cuando es inédito, paradójico, sorprendente, de interés público y actual, aunque haya ocurrido en un pasado remoto. Este hecho en cuanto es conocido por los periodistas, puede ser informado de manera oral por televisión, por radio, o de forma escrita en la prensa.

La noticia como texto posee una estructura. Las partes que la componen son el titular, el lead o entradilla, y el cuerpo (Alex Grijelmo, 2014). Tradicionalmente, la información se organiza jerárquicamente. En el lead, el primer párrafo, se resume la noticia, mientras que los detalles se desarrollan en el cuerpo, empezando 
siempre por los más importantes. Esta forma de organización responde al esquema de la pirámide invertida, aunque no es el único recurso.

Por otro lado, Yanes Mesa (2003) postula que los elementos que componen una noticia son los siguientes: el hecho principal, los comentarios (reacciones de los protagonistas directos o de las personas relacionadas al hecho) y los antecedentes (datos del pasado que se relacionan con el hecho principal).

\subsubsection{Los reportajes interpretativos}

Los reportajes o reportajes interpretativos pueden ampliar la información sobre un hecho que fue noticia, o un hecho actual, aunque no es una condición necesaria; puesto que también pueden abordar temas culturales, científicos, artísticos, o historias de interés humano (Correa Chávez, 2012).

En relación con su estructura, Paniagua Santamaría (2009) distingue cuatro componentes para el reportaje interpretativo: acontecimiento principal, antecedentes y contexto, reacciones e interpretaciones, y análisis valorativo. De acuerdo con el autor, el acontecimiento principal es el hecho noticioso o la noticia que motiva el reportaje. Los antecedentes y el contexto abarcan la información pasada y actual relacionada que permite contextualizar, o explicar el tema. Las reacciones e interpretaciones provienen de los protagonistas de la historia, de los testigos o de especialistas. Por último, el análisis valorativo constituye las proyecciones o consecuencias del hecho principal que se fundamentan en los hechos presentados.

\subsubsection{Los reportajes informativos}

Paniagua Santamaría (2009) sostiene que el reportaje informativo es un género intermedio, que se ubica entre la noticia y el reportaje propiamente dicho. Los reportajes informativos, de acuerdo con el autor, son relatos que se alejan de la noticia en cuanto incluyen mayor elementos interpretativos, pero que no llegan a ser reportajes porque estos elementos no derivan en una valoración de los hechos. Por ello, más que reportaje, el autor los considera relatos interpretativos.

\subsubsection{Las columnas de opinión}

Las columnas forman parte de los géneros de opinión. A través de las columnas, se expresa una evaluación subjetiva sobre los hechos actuales que han sido abordados en otras secciones del diario. 
El presente de subjuntivo con valor retrospectivo en la prensa peruana

Mabel Alelí Castro Yauri

Este género no posee una estructura fija; por tanto, el columnista tiene mayor libertad para elegir el modo en que brinda su punto de vista (Moreno Espinoza, 2007). Asimismo, el tono puede ser más ameno o conversacional a diferencia del editorial, género de opinión que se caracteriza por su alto grado de formalidad.

\subsection{La prensa peruana: tipos de diarios}

En la prensa peruana existe una distinción entre diarios chichas y diarios serios. Los primeros se enmarcan dentro del periodismo sensacionalista y poseen rasgos específicos. Para describir a los diarios chichas y distinguirlos de los diarios serios, Gargurevich Regal (1999) apela a dos aspectos: los temas y los recursos para captar la atención del público. Respecto al primero, el autor sostiene que el objetivo de los diarios chichas no apunta a brindar una mayor comprensión y profundización de los acontecimientos locales e internacionales. Por tanto, se deja de lado lo político, lo económico, lo cultural y se centran más bien en temas populares. Entre sus páginas abunda la información sobre personajes del mundo artístico, de programas de televisión o del deporte, y explotan las noticias violentas. Respecto a los recursos para promocionarse, se opta por el uso de colores llamativos en la primera plana, imágenes de mujeres desnudas o semidesnudas y titulares que emplean jergas criollas o del hampa, o que expresan un doble sentido.

Por otro lado, desde 1995, las empresas periodísticas peruanas han empezado a explorar el periodismo digital (Yezer'ska y Pozo, 2016). Este periodismo se caracteriza por difundir sus contenidos por internet tomando provecho de las herramientas de hipertextualidad, multimedialidad e interactividad que ofrece el ciberespacio (Salaverría, 2019). En el caso peruano, dentro de este campo, también se puede distinguir dos tipos de diarios: los tradicionales y los nativos digitales. Los primeros son los diarios que se trasladaron a la red, tales como El Comercio, La República, Perú21, etc. Los segundos son los diarios que nacieron en internet y que no ofrecen publicaciones físicas de sus contenidos. En este segundo bloque, nos referimos únicamente a las plataformas dirigidas por un equipo de periodistas. Ejemplo de ello son IDL-Reporteros, Ojo Público, Salud con Lupa, Corresponsal. pe o La Mula. 


\section{Metodología}

Este artículo presenta pate de los datos y el análisis que conforman la investigación de mi tesis sobre el presente de subjuntivo con valor retrospectivo desde un enfoque sociolingüístico.

El estudio es de tipo cuantitativo; el objetivo es cuantificar la frecuencia del uso del presente de subjuntivo y determinar la probabilidad de su ocurrencia según el registro y el género periodístico. Partiendo de la definición gradual del registro que describe Moreno Fernández (1998), establecimos un registro más formal, representado por los diarios digitales considerados serios, y uno menos formal, conformado por los diarios digitales chichas. Asimismo, en cuanto al género periodístico, este factor estuvo compuesto por cuatro categorías: las noticias, los reportajes informativos, los reportajes interpretativos y las columnas de opinión.

De otro lado, el recojo de los datos se dio entre noviembre del 2019 y enero del 2020. Se eligieron en total 150 textos periodísticos. 120 de ellos proceden de la prensa digital seria (El Comercio, Somos, La República, Gestión, Diario Correo, RPP Noticias, Perú21, IDL-Reporteros y Ojo Público). Los otros 30 fueron seleccionados de tres diarios digitales chichas (El Chino, El Trome y Ojo). Cada texto elegido debía tener como mínimo un caso del uso del presente de subjuntivo para referir a hechos pasados.

Conformado el corpus, se procedió a extraer todos los enunciados en los que el presente del subjuntivo y el pretérito imperfecto de subjuntivo alternaban. Los datos finales fueron evaluados con el programa estadístico Goldvarb X. Este software forma parte de los programas de regla variable que fueron diseñados con el objeto de realizar análisis sociolingüísticos (Sankoff et al., 2005; Tagliamonte, 2006). Con esta herramienta se puede realizar análisis probabilísticos de variantes lingüísticas.

\section{Análisis}

En esta sección, presentamos los resultados del análisis estadístico y su discusión. En primer lugar, se incluye el total de casos de variación, así como la distribución del presente de subjuntivo según cada factor independiente. En segundo lugar, se ubican los resultados del análisis probabilístico: el análisis binomial de subida y de bajada (binomial, up and down). Por último, en la discusión se interpretan los resultados. 


\subsection{Análisis descriptivo}

\subsubsection{Distribución total de las variantes}

La tabla 1 muestra el número total de casos del presente de subjuntivo y del pretérito imperfecto que fueron extraídos de los 150 textos periodísticos, entre diarios serios y chichas.

Tabla 1. Distribución total del presente y del pretérito imperfecto

\begin{tabular}{l|l|l}
\hline Variantes & N & \% \\
\hline Presente & 217 & $\mathbf{6 3}$ \\
\hline Pretérito imperfecto & 128 & 37 \\
\hline Total & 345 & 100 \\
\hline
\end{tabular}

Se observa que el total de ocurrencias de ambas variantes es 345. De este total, $217(63 \%)$ corresponden a la variante presente de subjuntivo y 128 al pretérito imperfecto (37\%). La diferencia se muestra a favor del presente de subjuntivo en toda la data.

\subsubsection{Distribución del presente de subjuntivo en relación con la variable género discursivo}

En esta investigación, la variable género discursivo alude a los cuatro géneros periodísticos que se identificaron en el corpus: las noticias, los reportajes informativos, los reportajes interpretativos y las columnas de opinión. En la tabla 2 se detalla el número de casos y los porcentajes del uso del presente de subjuntivo y del pretérito imperfecto según cada una de estas categorías. 
El presente de subjuntivo con valor retrospectivo en la prensa peruana

Mabel Alelí Castro Yauri

Tabla 2. Distribución del presente y del pretérito imperfecto según el género discursivo

\begin{tabular}{|c|c|c|c|c|c|c|}
\hline \multirow{2}{*}{ Género discursivo } & \multicolumn{2}{|c|}{ Presente } & \multicolumn{2}{|c|}{ Pretérito imperfecto } & \multicolumn{2}{|c|}{ Total } \\
\hline & $\mathbf{N}$ & $\%$ & $\mathbf{N}$ & $\%$ & $\mathbf{N}$ & $\%$ \\
\hline $\begin{array}{l}\text { Reportaje } \\
\text { informativo }\end{array}$ & 45 & 72.6 & 17 & 27.4 & 62 & 18.0 \\
\hline Noticia & 91 & 72.2 & 35 & 27.8 & 126 & 36.5 \\
\hline $\begin{array}{l}\text { Reportaje } \\
\text { interpretativo }\end{array}$ & 56 & 56.6 & 43 & 43.4 & 99 & 28.7 \\
\hline Columna & 25 & 43.1 & 33 & 56.9 & 58 & 16.8 \\
\hline Total & 217 & 62.9 & 128 & 37.1 & 345 & \\
\hline
\end{tabular}

De acuerdo con el cuadro, el total de ocurrencias de ambas variantes en las noticias suma 126 (36.5\%), 99 (28.7\%) en los reportajes interpretativos, 62 (18\%) en los reportajes informativos y 58 (16.8\%) en las columnas de opinión.

En relación con la distribución del uso del presente de subjuntivo en cada categoría, se evidencia que la ocurrencia de esta variante se ve favorecida de modo semejante en los reportajes informativos y en las noticias. De las 62 formas verbales que se contabilizaron en los reportajes informativos, 45 corresponden al presente de subjuntivo, lo cual representa el $72.6 \%$ del total. El porcentaje de la frecuencia del presente en las noticias es similar; de las 126 unidades recogidas, 91 corresponden al presente de subjuntivo, lo cual constituye el $72.2 \%$ del total.

Por otro lado, la frecuencia del presente es relativamente más baja en los reportajes interpretativos y en las columnas de opinión. En los reportajes interpretativos se contabilizó 56 formas verbales del presente. Esta cifra representa el $56.6 \%$ de la data (99 datos obtenidos). En las columnas de opinión, se identificaron solo 25 unidades, que representa el $43.1 \%$ de los datos recogidos en este género (48).

Un aspecto que se resalta del cuadro es que la preferencia por el presente de subjuntivo en lugar del pretérito imperfecto para referirse a hechos pasados es alta en casi todos los géneros. Los porcentajes superan el $50 \%$, a excepción de las columnas de opinión, donde la variante pretérito imperfecto tuvo mayor preferencia. Los datos porcentuales se pueden observar en la siguiente gráfica: 
El presente de subjuntivo con valor retrospectivo en la prensa peruana Mabel Alelí Castro Yauri

Gráfico 2. Representación gráfica de porcentajes de la variante presente según el género periodístico

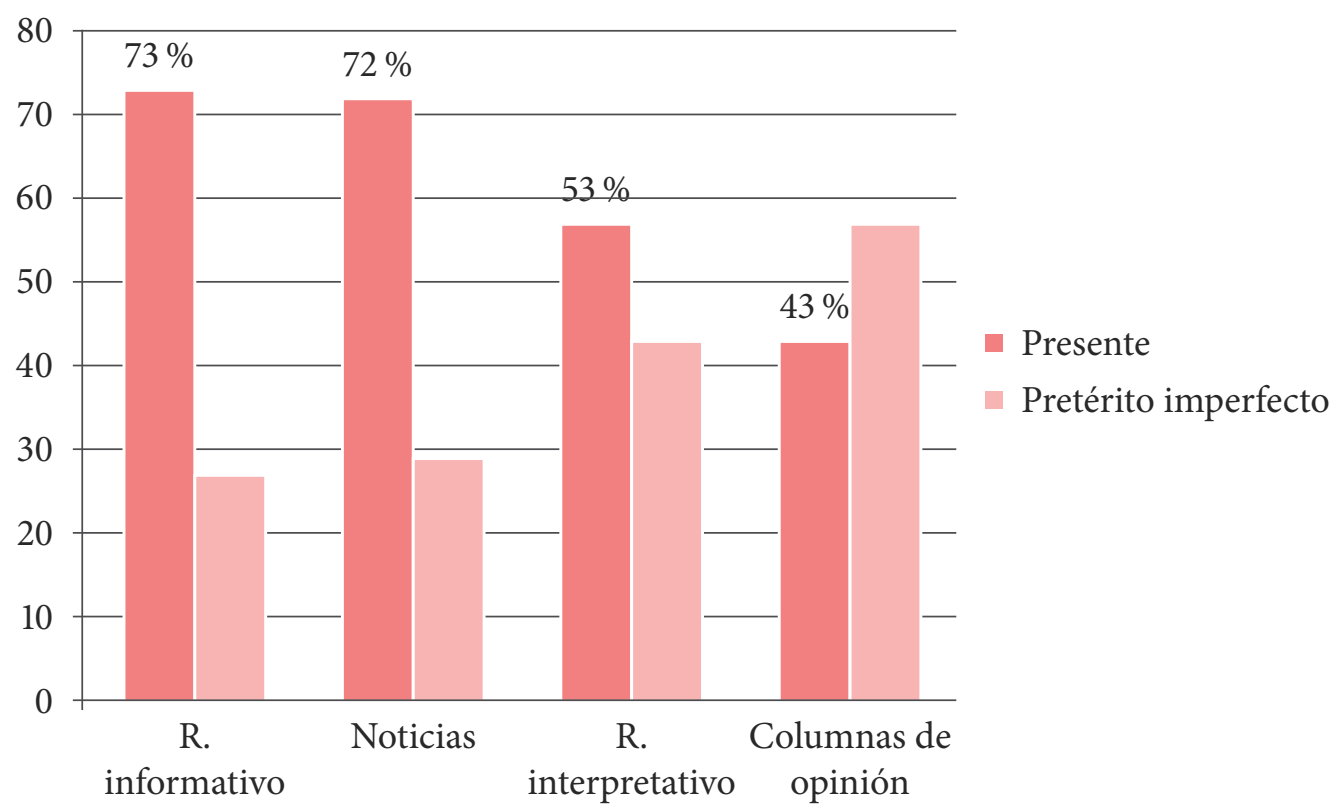

\subsubsection{Distribución del presente de subjuntivo en relación con la variable registro}

Para definir la variable registro, se tomó en consideración el tipo de diario. Los diarios serios constituyen el registro más formal y los diarios chichas, el registro menos formal. La tabla 3 muestra las cifras y los porcentajes de las variantes presente y pretérito imperfecto de acuerdo con estas categorías.

Tabla 3. Distribución del presente y del pretérito imperfecto según el registro

\begin{tabular}{l|l|l|l|l|l|l|l}
\multicolumn{1}{c|}{ Registro } & \multicolumn{3}{c}{ Presente } & \multicolumn{2}{c|}{ Pretérito imperfecto } & \multicolumn{2}{c}{ Total } \\
& N & \% & N & \% & N & $\%$ \\
\hline Más formal & 183 & 61.8 & 113 & $\mathbf{3 8 . 2}$ & 296 & 85.8 \\
\hline Menos formal & 34 & $\mathbf{6 9 . 4}$ & 15 & 30.6 & 49 & 14.2 \\
\hline Total & 217 & 62.9 & 128 & 37.1 & 345 & \\
\hline
\end{tabular}


La tabla 3 muestra que el total de ambas variantes en el registro más formal es $296(85.5 \%)$ y 49 (14.2 \%) en el registro menos formal. Esta diferencia responde al hecho de que se recabaron más textos de los diarios serios (120) que de los diarios chichas (30).

Por otro lado, en el cuadro se muestra que el porcentaje del uso del presente en un registro menos formal es $69.4 \%$, mientras que en un registro más formal equivale al $61.8 \%$. Sin embargo, la diferencia no es muy amplia. Por tanto, la elección del presente es mayor que la del pretérito en ambos registros.

\section{Gráfico 7. Representación gráfica de porcentajes de la variante presente según el registro}

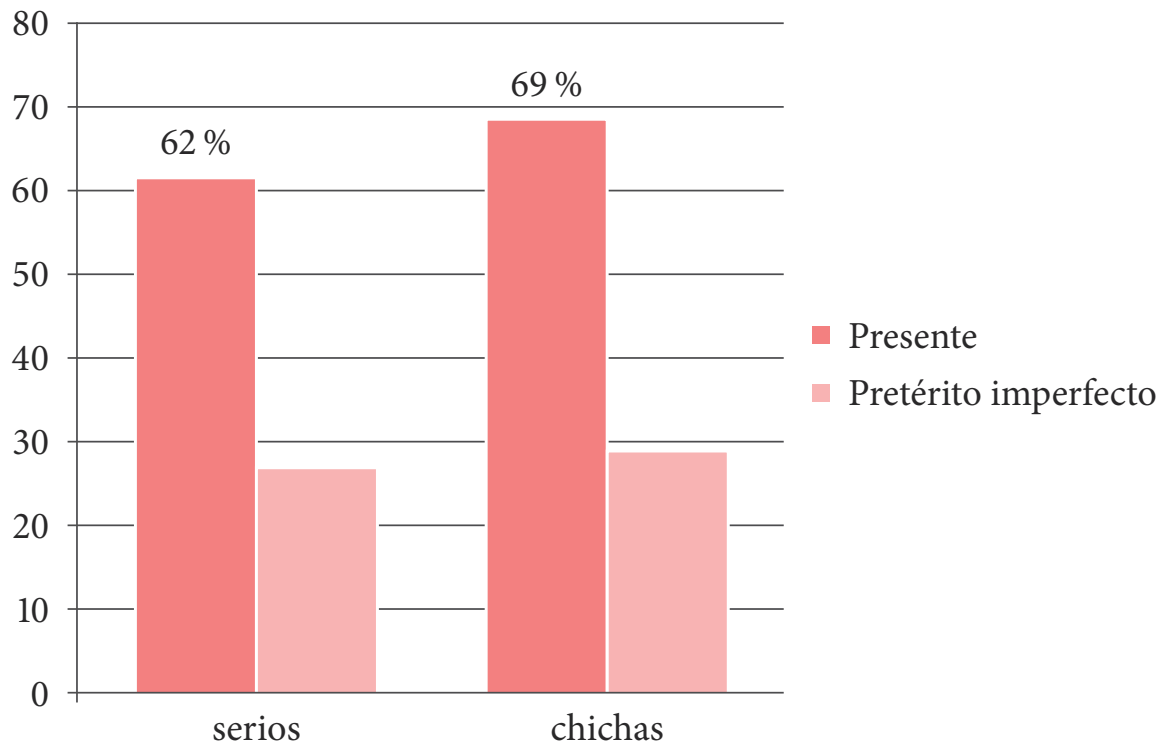

\subsection{Análisis probabilístico}

Antes de exponer los resultados de este segundo análisis, es necesario hacer algunas advertencias.

El resultado del análisis binomial de subida y de bajada que se presenta en esta sección, lo obtuve en la investigación de mi tesis Estudio sociolingüístico del uso del presente de subjuntivo en alternancia con el pretérito imperfecto de subjuntivo en los textos periodísticos de la prensa peruana (2020), en la cual estudié dos variables más: el género y la edad de los periodistas. Por tanto, los datos que se mostrarán 
El presente de subjuntivo con valor retrospectivo en la prensa peruana

Mabel Alelí Castro Yauri

en la tabla 4 formaron parte de un análisis que incluyó tales variables, aunque en este artículo mostramos los datos que son de nuestro interés.

Asimismo, en la tesis expliqué que, para el análisis probabilístico, no se pudo incluir la variable registro. Esto se debía a que el número de casos de la variable de estudio (presente/pretérito imperfecto) en los diarios chichas era inferior al de los diarios serios. Esta diferencia afectó la distribución de los datos en las tablas cruzadas. Se mostraban varias celdas vacías cuando los factores se intersectaban, lo cual significó un problema para el análisis probabilístico. Por lo tanto, los resultados que se muestran en la tabla 4 se basan en el total de variables que se recogieron en los diarios serios (296).

Ahora bien, al realizar la prueba, el programa estimó que solo un factor es significativo estadísticamente. Este factor es el género discursivo.

Tabla 4. Resultados del análisis de binomial de subida y de bajada.

\begin{tabular}{l|l|l|l}
\hline Género discursivo & Peso & $\%$ & N \\
\hline Reportaje informativo & $\mathbf{0 . 6 3}$ & 73.7 & $42 / 57$ \\
\hline Noticia & $\mathbf{0 . 6 1}$ & 72.1 & $62 / 86$ \\
\hline Reportaje interpretativo & 0.45 & 56.6 & $56 / 99$ \\
\hline Columna de opinión & 0.31 & 42.6 & $23 / 54$ \\
\hline $\begin{array}{l}\text { Media corregida }=0.619 \\
\text { Significación }=0.633\end{array}$ & \multicolumn{4}{|l}{ verosimilitud $=-188.370$} \\
\hline
\end{tabular}

En la parte inferior de la tabla 4 se observa que el valor de verosimilitud es -188.370. Este indicador permite establecer qué factor es significativo estadísticamente. El valor de verosimilitud debe ser lo más cercano a 0 para ser elegido por el programa. También, el análisis ofrece la media corregida (input). Este indicador está señalando la probabilidad de ocurrencia de la variante presente de subjuntivo en la data sin tener en consideración los factores independientes. La tabla señala que este valor es 0.619 , lo cual significa que hay una alta probabilidad del uso del presente en los diarios digitales peruanos.

De otro lado, la tabla incluye el peso probabilístico de cada categoría que compone este factor. El valor que aparece en cada caso está indicando qué género periodístico favorece o desfavorece el uso del presente de subjuntivo. En nuestro caso, son los reportajes informativos y las noticias los géneros que favorecen su 
ocurrencia, puesto que superan el valor requerido (0.5). El peso de los reportajes informativos equivale a $0.63 \mathrm{y}$ el de las noticias, 0.61 .

\subsection{Discusión}

Iniciamos este apartado haciendo referencia a los resultados del uso del presente en relación con el género discursivo. El análisis descriptivo de la muestra nos permitió ver que el presente de subjuntivo es empleado en lugar del imperfecto en los cuatro géneros periodísticos analizados. Sin embargo, la frecuencia en cada uno de ellos varía de manera significativa. El porcentaje en los reportajes informativos y en las noticias son los más altos: $74 \%$ y $72 \%$, respectivamente. Comparado con estos porcentajes, el de los reportajes interpretativos y el de las columnas de opinión son los más bajos: $57 \%$ y $43 \%$, respectivamente.

Esta diferencia guarda relación con el resultado del análisis probabilístico. El programa señaló que la probabilidad de ocurrencia del presente de subjuntivo en lugar del pretérito imperfecto incrementa o disminuye dependiendo de cada género periodístico. Esto indica que este factor es estadísticamente significativo, con lo cual cumplimos con el objetivo de determinar si había una correlación entre la variante presente y el género discursivo en los diarios digitales peruanos.

Respecto a los géneros que favorecen la variante, se halló que el presente de subjuntivo tiene una mayor probabilidad de emplearse en los reportajes informativos y en las noticias. La categoría más fuerte es el género reportaje informativo, su peso probabilístico es o.63 y, en segundo lugar, el género noticia, con un peso de 0.61 . En cambio, los pesos de los reportajes interpretativos y de las columnas de opinión, por debajo de 0.5, estarían indicando que, en los diarios digitales peruanos, estos tipos de textos son espacios que restringen el uso del presente, sobre todo las columnas de opinión.

El uso diferenciado del presente en estos cuatro géneros podría explicarse por dos factores. La primera posibilidad es el tiempo del que disponen los periodistas que redactan para diarios digitales y la segunda posibilidad es la importancia del tiempo presente en el periodismo.

En el primer caso, partimos del hecho de que las publicaciones de las notas periodísticas por internet son más inmediatas que las publicadas en los diarios impresos, sobre todo las noticias y los reportajes informativos. Robles Olivos (2018) señala que la presión por entregar este tipo de textos antes del cierre de edición es alta. En cambio, los reportajes interpretativos y columnas de opinión pueden ser desarrollados con mayor planificación, ya sea para los diarios impresos 
El presente de subjuntivo con valor retrospectivo en la prensa peruana

Mabel Alelí Castro Yauri

como digitales. Los reportajes interpretativos suelen ser el resultado de un trabajo prolongado (días, semanas, meses). Por ello, en los sitios web de los diarios abundan más las noticias y los reportajes informativos que los reportajes interpretativos. Las columnas de opinión también se publican una vez a la semana. Por tanto, el grado de planificación para redactar noticias y reportajes informativos es menor, más aún si estos se publican por internet.

En un estudio sobre la escrituralización de entrevistas publicadas en el diario digital El Comercio, Hernández Patrón (2017) señala que el factor tiempo puede ocasionar que lo periodistas filtren estructuras gramaticales que trasgreden la norma, aun cuando por lo general respeten sus reglas. Cabe advertir que, a partir de lo dicho por Hernández, no estamos afirmando que el uso del presente en lugar del imperfecto sea un error gramatical, sino más bien que este uso regional tan extendido pasa desapercibido en los textos periodísticos que conllevan un menor grado de planificación. De otro lado, recalcamos también que este tiempo de planificación es relativo. García García (2013) apunta que, a diferencia de algunos géneros dialógicos del internet, la escritura de los diarios digitales sigue procurando el cumplimento de la norma y tiene un mayor grado de planificación que las publicaciones de foros o chats.

En el segundo caso, consideramos también como segunda posibilidad que la importancia del tiempo presente en la redacción periodística puede explicar la diferencia del uso del presente de subjuntivo en cada género estudiado. Cuando Arrizabalaga (2009) analiza el modo en que se produjo el cambio temporal en las formas verbales del subjuntivo (presente/pretérito imperfecto), el autor menciona que el uso del presente para referir a hechos pasados ha prevalecido debido a que el cambio suele privilegiar la forma que expresa una mayor proximidad para el hablante. Inferimos que, para Arrizabalaga, el presente de subjuntivo expresa un sentido de proximidad sobre lo que se narra.

Este hecho es importante, puesto que, de acuerdo con Alex Grijelmo (2001), en el periodismo es recomendable utilizar el tiempo presente cuando se relatan hechos pasados con el objetivo de acercar los eventos al tiempo del lector. Gomis (1991) señala que esta es una regla para los titulares, aunque no se restringe solo a esta parte de los textos. Alex Grijelmo (2001) apunta que el presente histórico o el pretérito perfecto (he cantado) se convierten en recursos discursivos muy útiles para el relato de hechos remotos. En esta línea, por tanto, consideramos que el uso diferenciado del presente de subjuntivo en cada género estaría empleándose

160 Lengua y Sociedad. Revista de Lingüística Teórica y Aplicada 
como una estrategia discursiva que responde a la necesidad de actualizar o de aproximar los hechos pasados al tiempo del lector según se requiera en cada caso.

De otro lado, en relación con la variable registro, los datos obtenidos en el análisis mostraron que la frecuencia de la variante presente de subjuntivo fue alta en el registro menos formal (69\%) y baja en el más formal (62 \%). La diferencia, sin embargo, no es amplia. Además, la preferencia por el presente es alta en ambos registros. A partir de esta descripción, es posible que este factor no sea significativo estadísticamente. No obstante, es necesario un análisis probabilístico para confirmarlo. En la investigación no se pudo determinar si el registro influye en la elección del presente de subjuntivo en los diarios digitales peruanos.

Finalmente, un trabajo que contrasta con esta investigación es el de Adriana Speranza (2010). La autora analiza, desde un enfoque etnopragmático, los factores que se relacionan con la elección del presente de subjuntivo y del pretérito imperfecto en los textos de un diario argentino. Citamos su trabajo con el fin de comparar los datos identificados en aquella y en esta investigación.

En su estudio, Speranza recoge casos de variación (presente/pretérito imperfecto) en enunciados del discurso referido directo e indirecto. Esto significa que las oraciones subordinadas que analiza son únicamente nominales completivas en función de objeto directo. Esto podría indicar que en los diarios argentinos la extensión de la variación se restringe a este contexto sintáctico. La data recogida en los diarios digitales peruanos, por el contrario, muestra que la variación no solo se presenta en este tipo de oraciones, sino también en oraciones finales, relativas, temporales y otros tipos de oraciones nominales completivas. Además, estas oraciones subordinadas no solo expresan lo dicho por otra persona, sino también describen situaciones dentro de la narración periodística, como se observa en los siguientes ejemplos:

a. Una vez dentro de la agencia bancaria, el policía le dijo desafiante: «Tienes que darle su cariño al comandante. La chamba la hemos hecho todos», y le entregó su canguro para que guarde ahí el dinero (Ana Briceño, 2019).

b. Esa misma tarde, por orden del Ministerio de la Mujer y al considerar que no tenía familiares que puedan cuidar de ella, Nicole fue enviada a la Casa Hogar de la Niña Belén, una institución administrada por la Beneficencia Pública que acoge a menores infractores y en abandono en la ciudad de Cajamarca. (Salazar Vega, 2019). 
El presente de subjuntivo con valor retrospectivo en la prensa peruana

Mabel Alelí Castro Yauri

c. El 16 de febrero del 2017, un día antes que viajen para la inauguración del Santuario Ecológico de Lloqllapampa, en memoria de las víctimas, Jesús Cossio entregó los retratos a los deudos. (Castro Cajahuanca, 2017).

\section{Conclusiones}

De acuerdo con la investigación, se observó que la variable género discursivo influye en la ocurrencia del presente de subjuntivo en los diarios digitales peruanos. El análisis probabilístico señaló que esta variación es significativa estadísticamente. La variante no estándar se ve favorecida principalmente en los reportajes informativos y en las noticias. La probabilidad de su elección es mayor en ambos géneros de modo semejante. En cambio, la probabilidad disminuye en los reportajes interpretativos y en las columnas de opinión. Estos últimos textos constituyen el género que constriñe principalmente a la variante. La preferencia del presente en los reportajes informativos y en las noticias podría atribuirse al menor tiempo que implica la redacción de las noticias y de los reportajes informativos en los diarios digitales. Aunque no se puede descartar, por otro lado, la posibilidad de que el presente con valor retrospectivo esté siendo empleado como un recurso discursivo que permite actualizar los hechos pasados al tiempo del lector. En tal caso, la diferencia respondería a la necesidad de actualización en cada género.

Por otro lado, en relación con el factor registro, no se pudo determinar si esta variable es significativa estadísticamente. Se observó en la distribución de la data que la diferencia del uso del presente en ambos registros no es tan amplia, lo cual podría dar un indicio de que el factor no influye en la elección de la variante innovadora. Sin embargo, para confirmarlo, es necesario incluir mayores datos procedentes de los diarios chichas. Por otro lado, resaltamos el hecho de que la probabilidad de ocurrencia del presente de subjuntivo con valor respectivo sea alta en los diarios digitales serios. El análisis probabilístico indica que en estos diarios, por cada 100 casos de variación presente/pretérito imperfecto, 61 corresponden al presente. Esta información evidencia la extensión de la variante no estándar en un tipo de comunicación formal.

El estudio del presente de subjuntivo en los diarios digitales peruanos aporta conocimiento sobre el fenómeno de variación presente/pretérito imperfecto en el español peruano. Desde un enfoque cuantitativo, se ha podido confirmar que la elección del presente en detrimento del pretérito imperfecto es alta en los medios 
periodísticos peruanos; por lo tanto, la variable constituida por ambas variantes no se restringe, en el español peruano, al habla popular o a los contextos espontáneos. Asimismo, el estudio brinda información sobre el desarrollo del presente de subjuntivo en la prensa peruana. El tipo de oraciones en el que ocurre la variable no se restringe a las subordinadas nominales en complemento de un verbo de habla; tampoco se presenta únicamente dentro de un discurso referido, sino también en enunciados que describen situaciones. En ese sentido contrasta con los casos que reporta Speranza (2010) de la prensa argentina.

Por último, es necesario realizar mayores estudios para determinar si el uso del presente de subjuntivo con valor retrospectivo forma parte de la norma del español peruano. La investigación se enfocó en las producciones escritas formales de los periodistas, pero se debe considerar también otras poblaciones, así como seguir evaluando la significatividad de la variable registro.

\section{Referencias bibliográficas}

Arrizabalaga Lizarraga, C. (2009). «Imploraban que no lo maten». Reorganización de los tiempos del subjuntivo en español peruano. Moenia, 15, 295-311. http:// hdl.handle.net/10347/5664

Bawarchi, A. S. y Reiff, M. J. (2010). Genre. An Introduction to History, Theory, Research, and Pedagogy. Parlor. https://wac.colostate.edu/books/ referenceguides/bawarshi-reiff

Briceño, A. (2019, 11 de junio). Recompensas fallidas: programa del Mininter y miles de soles bajo la lupa. El Comercio. https:/elcomercio.pe/lima/seguridad/ recompensas-fallidas-programa-mininter-miles-soles-lupa-informe-video-noticia-ecpm-642908-noticia

Castro, J. (2017, 20 de agosto). Accomarca: Retratos contra el olvido. Ojo Público. https://memoria.ojo-publico.com/articulo/accomarca-retratos-contra-elolvido

Castro Yauri, M. A. (2020). Estudio sociolingüístico del uso del presente de subjuntivo en alternancia con el pretérito imperfecto de subjuntivo en los textos periodísticos de la prensa peruana [Tesis de licenciatura, Universidad Nacional Mayor de San Marcos]. Cybertesis. https://hdl.handle.net/20.500.12672/16084

Correa Chávez, M. J.(2012). El reportaje en el ciberperiodismo [Tesis de Licenciatura, Universidad de Piura]. Repositorio Institucional Pirhua. https://pirhua.udep. edu.pe/handle/11042/1720 
El presente de subjuntivo con valor retrospectivo en la prensa peruana

Mabel Alelí Castro Yauri

García García, E. (2013). La puntuación en la prensa digital [Tesis de Maestría, Universitat de Barcelona]. Repòsit Digital de la Universitat de Barcelona. http:// diposit.ub.edu/dspace/handle/2445/47065

Gargurevich Regal, J. (1999). Lo Real/Exagerado La prensa sensacionalista en el Perú De las Relaciones a los Diarios Chicha [Tesis de Maestría, Pontificia Universidad Católica del Perú]. Repositorio institucional de la Pontificia Universidad Católica del Perú. http://tesis.pucp.edu.pe/repositorio/handle/20.500.12404/4534

Gomis Sanahuja, L. (1991). Teoría del periodismo. Cómo se forma el presente. Paidós.

Grijelmo, A. (2001). El estilo del periodista (8. $\left.{ }^{\mathrm{a}} \mathrm{ed}\right)$. Taurus.

Grijelmo, A. (2014). El estilo del periodista (18. ${ }^{\mathrm{a}}$ ed.). Taurus.

Hernández Patrón, U. I. (2017). El paso del medio fónico al medio gráfico: análisis sintáctico de la escrituralización de noticias en la publicación digital del diario El Comercio [Tesis de Maestría, Pontificia Universidad Católica del Perú]. Repositorio institucional de la Pontificia Universidad Católica del Perú. http:// tesis.pucp.edu.pe/repositorio/handle/20.500.12404/8825

Martín-Martín, P. A. (2003). Genre and discourse community. Revista de filología inglesa, 25, 153-156. http://uvadoc.uva.es/handle/10324/17297

Mejía Chiang, C. (2012). Géneros y estilo de redacción en la prensa. Desarrollo y variantes taxonómicas. Correspondencias \& Análisis, 2, 201-2017. doi: 10.24265/ cian.2012.n2.10

Moreno Espinosa, P. (2007). Opinión y géneros en el periodismo electrónico: redacción y escritura. Ámbitos, 16, 123-149. https://www.redalyc.org/articulo. oa? id $=16801608$

Moreno Fernández, F. (1998). Principios de sociolingüística y sociología del lenguaje. Ariel.

Paniagua Santamaría, P. (2009). Información e interpretación en periodismo. Hacia una nueva teoría de los géneros. voc.

RAE. (2010). Manual de la Nueva gramática de la lengua española. Espasa.

Robles Olivos, R. A. (2018). Prensa gratuita en el Perú y su impacto en la formción de nuevos lectores de medios impresos en jóvenes residentes de Lima entre 15 a 25 años. Estudio de caso: Publimetro (Agosto de 2017 - enero de 2018) [Tesis de maestría, Universidad San Martín de Porres]. Repositorio Académico de la Universidad San Martín de Porres. https://repositorio.usmp.edu.pe/ handle/20.500.12727/4111

164 Lengua y Sociedad. Revista de Lingüística Teórica y Aplicada 
Rojo Sánchez, G. y Veiga Rodríguez, A. (1999). El tiempo verbal. Los tiempos simples. En I. Bosque y V. Demonte (Eds.), Gramática descriptiva de la lengua española (Vol. 2, pp. 2867-1934). Espasa.

Salaverría, R. (2019). Periodismo digital: 25 años de investigación. Artículo de revisión. El profesional de la información, 28(1), 1-27. https://recyt.fecyt.es/ index.php/EPI/article/view/69729

Salazar Vega, E. (2019, 22 de octubre). Abortar en Perú: Cuando víctima y familiares son llevados a la cárcel. Ojo Público. https://ojo-publico.com/1411/ abortar-en-peru-victima-y-familiares-son-llevados-carcel

Sankoff, D., Tagliamonte, S., y Smith, E. (2005). Goldvarb X: A variable rule application for Macintosh and Windows [Softaware]. Universidad de Toronto y Universidad de Ottawa. http://individual.utoronto.ca/tagliamonte/goldvarb. html

Sessarego, S. (2008). Spanish Concordantia Temporum: An Old Issue, New Solutions. En M. Westmoreland y J. Thomas (Eds.), Selected Proceedings of the 4th Workshop on Spanish Sociolinguistics (pp. 91-99). Cascadilla Proceedings Project. http://www.lingref.com/cpp/wss/4/index.html

Speranza, A. A. (2010, abril). "Nos gritaron que nos fuéramos» (¿o que nos vayamos?) El uso variable de los tiempos verbales como estrategia etnopragmática. Artículo presentado en el IX Congreso Argentino de Hispanitas, La Plata. http://sedici.unlp.edu.ar/handle/10915/39303

Tagliamonte, S. A. (2006). Analysing Socioloinguistic Variation. Universidad de Cambridge. https://doi.org/10.1017/Сво9780511801624

Veiga Rodríguez, A. (1996). Subjuntivo, irrealidad y oposiciones temporales en español. En G. Wotjak (Ed.), El verbo español. Aspectos morfosintácticos, sociolingüísticos y lexicogenéticos (pp. 40-71). Iberoamericana.

Veiga Rodríguez, A. (2014). ¿Por qué pretérito imperfecto de subjuntivo? Liburna, 17, 99-116. https://dialnet.unirioja.es/servlet/articulo? codigo $=5141974$

Vellón Lahoz, J. (2011). El registro coloquial en el texto informativo: entre el estilo periodístico y la estrategia comunicativa. Estudios sobre el mensaje periodístico, 17(2), 675-69o. https://revistas.ucm.es/index.php/ESMP/article/view/38138

Yanes Mesa, R. (2003). La noticia y la entrevista. Aproximación a su concepto y estructura. Ámbitos, 9-10, 239-272. https://dialnet.unirioja.es/servlet/articulo? codigo $=793087$

Yezers'ka, Lyudmyla y Pozo, R. (2016). Veinte años de medios digitales en el Perú (1995-2015). Universidad de Piura. 
El presente de subjuntivo con valor retrospectivo en la prensa peruana

Mabel Alelí Castro Yauri

\section{Trayectoria académica de la autora}

Mabel Alelí Castro Yauri es licenciada en Lingüística. Forma parte del grupo de investigación Lenguas y Filosofías del Perú de la Facultad de Letras y Ciencias Humanas de la Universidad Nacional Mayor de San Marcos. Actualmente, cursa la maestría de Lingüística en esta casa de estudios. Sus temas de interés en lingüústica están relacionados con el discurso y su aplicación en la educación. 